\title{
D'un habitat mobile à un habitat fixe
}

Fondements et changements de l'orientationdans l'espace domestique mongol

From a mobile to a fixed dwelling. Foundations and changes of orientation within the Mongol domestic space

\section{Alexandra Marois}

\section{(2) OpenEdition}

\section{Journals}

Édition électronique

URL : https://journals.openedition.org/emscat/759

DOI : 10.4000/emscat.759

ISSN : 2101-0013

Éditeur

Centre d'Etudes Mongoles \& Sibériennes / École Pratique des Hautes Études

Édition imprimée

Date de publication : 1 mai 2006

Pagination : 207-237

ISBN : 2-9518888-3-X

ISSN : 0766-5075

Référence électronique

Alexandra Marois, « D'un habitat mobile à un habitat fixe », Études mongoles et sibériennes,

centrasiatiques et tibétaines [En ligne], 36-37 | 2006, mis en ligne le 17 mars 2009, consulté le 13 juillet 2021. URL : http://journals.openedition.org/emscat/759; DOI : https://doi.org/10.4000/emscat.759

Ce document a été généré automatiquement le 13 juillet 2021.

(c) Tous droits réservés 


\title{
D'un habitat mobile à un habitat fixe
}

\author{
Fondements et changements de l'orientationdans l'espace domestique \\ mongol
}

From a mobile to a fixed dwelling. Foundations and changes of orientation

within the Mongol domestic space

Alexandra Marois

\section{NOTE DE L'AUTEUR}

Je tiensà remercier Françoise Aubin et Roberte Hamayon pour les précieuses corrections et suggestions qu'elles ont apportées à ce texte. [Note du rédacteur en chef : le présent article est publié dans EMSCAT 36-37, sous la responsabilité scientifique de $\mathrm{M}^{\mathrm{mes}} \mathrm{F}$. Aubin et R. Hamayon.]

Dans les steppes de Mongolie-Intérieure, parmi les campements nomades, on assiste ces dernières années à la multiplication de bâtiments fixes dispersés dans le paysage. Ils sont habités temporairement par des familles d'éleveurs qui vivaient jusqu'ici toute l'année sous la yourte ${ }^{1}$. La cohabitation et l'utilisation alternée de ces deux formes d'habitat offrent l'occasion de comparer les façons dont un même groupe s'approprie des espaces domestiques différents. Par l'étude du passage de la tente circulaire et mobile à la maison angulaire et fixe, nous allons voir comment et par quels procédés les éleveurs transposent, adaptent et transforment leurs manières d'habiter. À travers les changements d'orientation dans l'espace domestique, nous tenterons d'isoler les divers paramètres qui guident les manières de choisir un sens.

2 La question de l'orientation de l'habitat mobile a fait l'objet de nombreuses études anthropologiques. Le cas des pasteurs mongols constitue néanmoins un exemple singulier et révélateurdans la mesure où, non seulement à travers les siècles, mais aussi sur tout le territoire qu'ils occupent, ils ont conservé des manières quasiment 
inchangées de placer et d'organiser leur habitat. D'une yourte à l'autre, les meubles et les objets de la vie courante sont agencés de manière similaire. De plus, les positions qu'occupent, sous la yourte, les habitants et les invités répondent à des codes partagés par tous les Mongols. Cependant, malgré cette apparente continuité dans le temps et dans l'espace, notre expérience du terrain, en tant que chercheur et en tant que "maîtresse de yourte", nous a permis d'identifier des changements et surtout des ajustements quotidiens sur lesquels nous souhaitons focaliser notre attention.

À partir du cas de la bannière de gauche des Nouveaux Barga en Mongolie-Intérieure ${ }^{2}$ - zone de steppe peuplée d'éleveurs pratiquant majoritairement le nomadisme pastoral - nous procéderons à une comparaison dont le propos n'est pas d'opposer la yourte au bâtiment, mais de mettre en évidence les continuités et les ruptures dans le passage d'un mode d'habitat à l'autre.

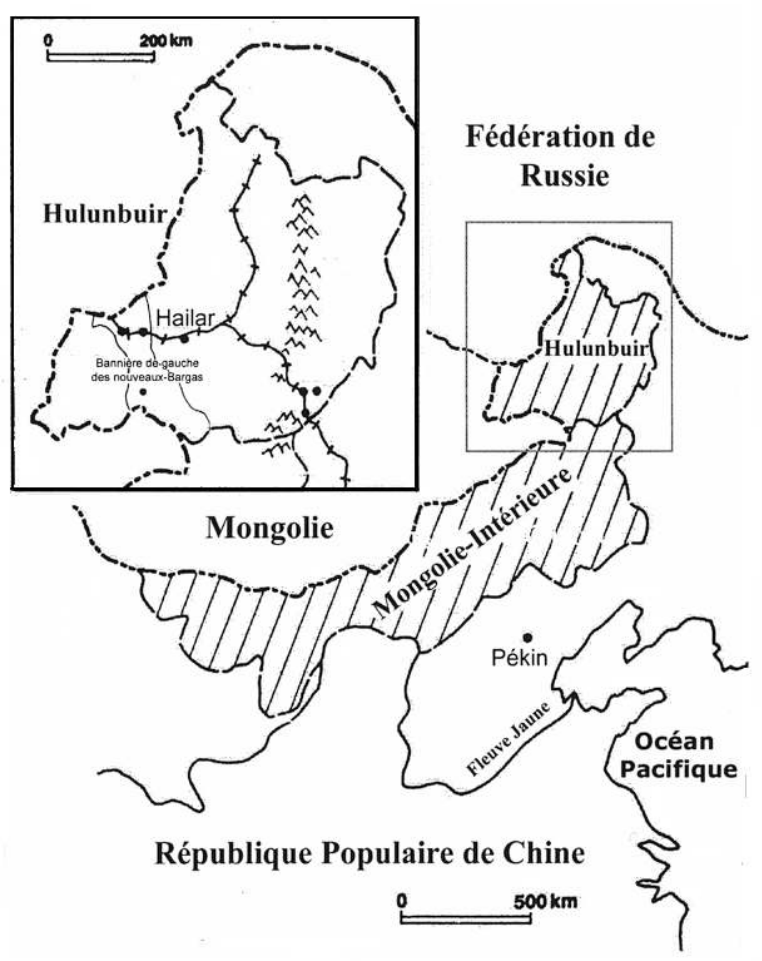

$4 \quad$ Une description détaillée de ces deux types de résidence nous permettra de saisir dans quelle mesure la disposition et l'orientation des objets participent au quotidien d'un positionnement variable des familles et des individus au sein de l'espace social. Elle montrera aussi que le recours aux points cardinaux, repère classique de l'orientation occidentale, ne suffit pas à éclairer la question de l'orientation dans l'espace intérieur. Certes, les points cardinaux sont utiles à la description, mais ils fixent de manière excessive des états qui nécessitent un regard plus souple pour une compréhension affinée de l'habitat. En outre, l'analyse comparative révélera le rôle décisif du foyer et du visiteur dans la disposition des objets et des individus.. Enfin, nous mettrons en évidence des changements dans les rapports à l'environnement, vécus et perçus différemment en fonction de l'habitat occupé. 


\section{La yourte}

\section{Composantes, formes et matériaux}

5 La tente ronde qu'habitent les éleveurs nomades est appelée isegei ger en mongol littéraire. Le terme générique ger, s'utilise pour rendre les notions d'« étui », de " housse ", en fait de tout ce qui enveloppe et protège. Le terme isegei qui le détermine, signifie « feutre ", matière qui compose la couverture de l'habitat. « Ce qui enveloppe et protège en feutre » est donc la formule utilisée quotidiennement par les Mongols pour désigner leur tente.

6 Dans le discours, le choix de la forme circulaire trouve sa justification dans le caractère extrêmement venté du climat local : elle ne donne pas prise au vent. Mais, audelà de cet aspect technique, ses habitants lui confèrent aussi une valeur esthétique : elle s'harmoniserait à leur environnement. Cet espace unique et circulaire engendre un agencement particulier du mobilier: il impose une continuité, les meubles se juxtaposant les uns aux autres. Il conditionne aussi une façon particulière de se mouvoir et permet la réunion en un même lieu de tous les membres de la maisonnée, une promiscuité qui assure une cohésion entre les habitants. L'intimité partagée a pour conséquence une profonde connaissance de l'autre et peut être pensée comme une forme de lien social.

7 Les yourtes que l'on rencontre en pays barga sont relativement petites en comparaison de celles de Mongolie. D'une hauteur allant de deux mètres à deux mètres cinquante pour la partie centrale, elles ne sont, pour la plupart, composées que de quatre treillis muraux, qana, contre cinq en moyenne en Mongolie. L'« anneau de compression " ou toyunu, estfait en pin ou encore en métal, matériau qui tend à se répandre de plus en plus.

8 Tous les éléments en bois sont peints en bleu azur ou en orange; seuls les nouveauxanneaux en métal comportent du rouge, du bleu foncé et du vert ${ }^{3}$. Les vieilles armatures ont pour la plupart perdu leurs teintes, mais le caractère coloré est primordial car les éleveurs considèrent qu'une yourte neuve en bois brut est inachevée.

9 Sur l'armature en bois vient se poser le revêtement de la yourte fait de feutre, isegei, matériau obtenu en foulant ou en agglutinant du poil ou de la laine. Dans les années cinquante et soixante du vingtième siècle, il était encore confectionné au sein de la famille, mais actuellement il est acheté dans les villages auprès d'artisans spécialisés, lesquels sont souvent patronnés par un $\mathrm{Han}^{4}$.Cependant, on continue, chaque printemps, à le réparer et à le rapiécer en famille - vestige d'une activité majeure qui procure l'occasion de transmettre une partie d'un savoir-faire lié à la yourte.

10 La plupart du temps, le sol est laissé tel quel, et seule la partie entre le poêle central et les meubles du fond, soit le secteur honorifique, qojmar ${ }^{5}$, est recouvert d'une couche de feutre ou de tissu épais. Ce revêtement peut s'étendre jusqu'aux pieds des lits, mais jamais jusqu'à l'entrée, de l'autre côté du poêle. À l'approche de l'hiver, certaines familles recouvrent le sol de briques ${ }^{6}$ pour isoler l'intérieur de la yourte du froid. Ce revêtement contourne le mobilier déjà installé, occupe toute la surface praticable et comporte parfois une ouverture donnant sur une niche creusée dans le sol où sont conservés pommes de terre et légumes consommés en hiver. Ce parterre en briques est laissé en place quand on nomadise; il laisse sur le sol une marque qui dessine la 
configuration interne de la yourte. On observe ainsi, dispersées à travers la steppe, des traces durables d'anciens campements d'hiver. Chez les couples récemment mariés ou chez les familles riches, on voit de plus en plus apparaitre des parquets qui recouvrent la totalité de la surface intérieure. Ils sont tous peints de couleur orange, de même que les perches du toit.

11 La porte, egüde $e^{7}$, se compose d'un chambranle et d'un battant en bois ${ }^{8}$, articulés par des charnières de cuir ou parfois de bois. Elles se trouvent toujours sur le côté gauche ${ }^{9}$ en entrant et ouvrent vers l'extérieur. Il y a quelques années, les portes ne se fermaient que de l'intérieur. Il était en effet très rare qu'un campement reste totalement sans habitants et, en cas d'absence, on comptait sur les chiens pour empêcher les intrus d'entrer. Les portes neuves sont maintenant toutes munies d'une attache de cadenas à l'extérieur. Certaines possèdent aussi des fenêtres dans leur partie supérieure ; comme le verre résiste mal aux claquements de porte et aux déplacements saisonniers, il est parfois remplacé par une mince couche de bâche plastique. On trouve par ailleurs d'autres modèles avec deux battants en bois dont les parties supérieures comportent elles aussi des ouvertures; elles procurent une plus grande luminosité au sein de l'habitat et permettent en outre d'observer ce qui se passe à l'extérieur ${ }^{10}$, question sur laquelle nous allons revenir. Pour les éleveurs de la région Barga, caractérisée par ses étendues planes parsemées de lacs, il faut orienter la porte vers le sud ou mieux, le sudouest. En pratique, les portes sont intuitivement dirigées vers le sud, le sud-est ou le sud-ouest; le seul ajustement remarquable consiste à adapter la position en fonction de la pente du terrain.

\section{Les variations dans l'orientation : manipulations identitaires}

12 Il est généralement admis que, à l'origine, la porte des yourtes d'Asie Centrale et d'Asie Intérieure était orientée en direction de l'est et que plus tard, elle l'aurait été vers le sud, sauf chez les Ordos qui auraient maintenu une ouverture à l'est (Mostaert 1968, p. 7). Toutefois, une étude menée par Kotwicz (1929) couvrant l'aire centreasiatique, montre que même dans des temps très anciens, on trouve des orientations vers le sud et vers l'ouest.

13 Le choix d'une orientation vers l'est est généralement justifié par les vents dominants qui viennent $\mathrm{du}$ nord-ouest et par les croyances religieuses (bouddhiques ou chamaniques) qui attribuent à l'est, origine du soleil levant, un caractère faste. Quant à l'adoption d'une orientation en direction du sud, les interprétations courantes y voient l'effet d'une influence chinoise ${ }^{11}$, ou bouddhiste peut-être (Charleux 1997, p. 211). D'un point de vue climatologique, une ouverture au sud est considérée localement comme protégeant mieux des vents dominants venant de Sibérie.

14 Cependant, ces hypothèses ne permettent pas d'apporter une explication suffisante au changement d'orientation de l'est vers le sud. Peut-on imaginer qu'une communauté d'éleveurs nomades décide dans son ensemble de modifier l'orientation de sa porte sous l'effet de nouvelles croyances, sans tenir compte des conditions environnementales? Nous pensons plutôt qu'un ensemble de facteurs influe sur de tels choix. Liés à la configuration de l'espace local et à l'adoption de nouveaux systèmes religieux, ces choix peuvent dépendre aussi de manipulations variables en fonction du rapport des individus entre eux et avec la communauté plus large dans laquelle ils s'insèrent. L'analyse proposée par Uradyn Bulag, que confortent nos propres 
matériaux, contribue à mettre en évidence l'intervention de cet autre paramètre, de nature idéologique ou plus précisément identitaire.

15 U. Bulag (1998, p. 177), dans son étude des symboles nationaux mongols, montre que la Mongolie, en tant que lieu d'origine de tous les Mongols, est depuis longtemps ${ }^{12}$ perçue comme une terre sacrée, comparable à la « Mecque » par les formes d'allégeance symbolique qu'on lui prête. Il illustre cette hypothèse par l'exemple, entre autres, du musée de Mongolie-Intérieure à Kökeqota (Hohhot) - sa capitale - sur le toit duquel, au moment de sa construction en 1957, fut installée la statue d'un cheval volant, orientée vers le nord, c'est-à-dire vers la République de Mongolie. Durant la révolution culturelle, alors que se développaient des mouvements sécessionnistes dans les régions mongoles, les autorités chinoises s'opposèrent à ce symbole dirigé vers l'extérieur, y décryptant un puissant message politique de la part des Mongols de MongolieIntérieure. L'édifice fut donc détruit et une nouvelle statue reconstruite, cette fois face au sud, de manière à réorienter symboliquement la minorité mongole vers le cœur du territoire chinois ${ }^{13}$.

À cet exemple particulièrement évocateur, l'auteur ajoute que, dans de nombreuses yourtes du Barga, les éleveurs posent leurs selles à l'ouest, alors que les Oirates la placent à l'est et les Bouriates au sud, chacun en direction du cœur de sa terre ancestrale ${ }^{14}$. Par-là, $U$. Bulag met en avant le caractère symbolique des manipulations de l'orientation et montre que la manière de se positionner dans l'espace peut être aussi une façon de se définir politiquement vis-à-vis de l'État dominant.

17 Pour notre part, au cours d'une recherche en Mongolie dans le district de Hulunbuir ${ }^{15}$ (division administrative créée en 1945 pour accueillir les éleveurs ayant fui la Chine, dont le nom a été repris de la ligue de Mongolie-Intérieure d'où ils provenaient), nous avons observé une pratique tout à fait similaire. Un éleveur nous invita un jour sous sa yourte et au cours de la discussion, il nous apprit l'existence d'un autre groupe Barga établi au nord-est, à quelques centaines de kilomètres de là. Pris de mélancolie, il nous expliqua que parfois, pour se sentir plus proche des membres de sa communauté, il disposait sa selle dans leur direction, soit dans la partie nord-est de la yourte, pour symboliser le lien qu'il tient à entretenir avec eux.

18 Ces différents exemples incitent à s'interroger à nouveau sur les motivations liées au choix de l'orientation de la porte. L'observation montre que le paysage, qu'il soit de plaines, de lacs, de rivières ou encore de montagnes, influe sur le choix de son orientation, mais dans les cas dépourvus de contraintes géographiques, comme ceux auxquels nous sommes ici confrontée, un facteur symbolique peut intervenir. Ainsi pour un Barga, dire qu'il vaut mieux se tourner vers le sud-ouest que vers le sud-est signifie qu'il préfère se tourner vers les Mongols, plutôt que vers les Chinois han, situés dans ce cas précisément à l'est. Le choix d'une orientation particulière dépend donc de paramètres et de motivations variables selon les circonstances. Dans certain cas, il peut être lu comme l'expression d'une inclination personnelle ou communautaire, une forme de positionnement identitaire.

\section{Organisation intérieure}

19 Les Mongols utilisent un corpus précis de termes pour désigner les différents secteurs de la yourte. Au cours de la description qui va suivre, nous nous référerons 
exclusivement à ces termes pour aborder ensuite la question de l'orientation par rapport aux points cardinaux.



20 Dans la partie barayun uridu, juste après la porte, se trouvent les réserves de combustible ${ }^{16}$, directement posées sur le sol ou stockées dans une large caisse en métal. À côté, sur un trépied en métal ou au sol, est disposée la cuvette de toilette familiale. Juste derrière, est rangé tout ce qui est relatif au harnachement du cheval: selle, étriers, couvertures. En été et en automne, un espace est dégagé pour disposer la marmite dans laquelle repose la crème et au-dessus, sur le treillis, l'outre ${ }^{17}$ est accrochée.

21 Dans le secteur barayun, il y a généralement un lit réservé à l'hôte de passage mais pouvant être occupé en temps ordinaire par les membres de la famille. Lorsque la famille n'héberge aucun étranger, ce sont en priorité les cadets qui y dorment, les aînés s'installant sur le sol, au fond, au-delà du foyer. En effet, ces derniers préfèrent éviter aux enfants d'être exposés au froid venant du sol et de l'interstice entre la terre et le feutre de la yourte ${ }^{18}$. Par ailleurs, le fait de dormir sur le sol permet une meilleure écoute des bruits extérieurs, du bétail en particulier, auxquels les personnes âgées accordent en général une grande attention. Dans les rares cas où il n'y a pas de lit dans cette partie de la yourte, on y dispose des tabourets, une table ou d'autres objets susceptibles d'être fréquemment déplacés. Le visiteur s'assoit alors directement sur le sol ou sur un petit tabouret.

22 Vers le fond, qojtu, débute le secteur honorifique de la yourte, le qojmar. C'est dans cette zone que l'on invite l'hôte d'exception à s'asseoir. Derrière lui se trouvent deux ou trois meubles accolés les uns aux autres dans lesquels sont rangés les vêtements et les étoffes précieuses (on y cache aussi les trésors de famille : jumelles, bijoux, blagues à tabac, sucre, etc.). Sur ces commodes ou ces coffres sont empilées des valises contenant d'autres vêtements et tissus ainsi que des documents personnels. On y place également 
la machine à coudre et tout un ensemble d'objets «prestigieux». Alors qu'avant la répression maoïste s'y trouvait l'autel religieux, aujourd'hui y sont exposés essentiellement des petits jouets colorés en plastique, des bibelots, des horloges ${ }^{19}$, des lunettes, des radios, des miroirs, des produits de beauté et les photos de famille qui rappellent les ongon ${ }^{20}$. Les membres de la famille posent là les objets qu'ils sont fiers de posséder, ceux qu'ils considèrent être des signes de richesse ou de modernité, même si les plus précieux restent dissimulés dans les coffres à vêtements. De la même manière, quand une famille traverse une période difficile, il arrive que la viande ou la farine soient mises dans le fond de la yourte, car ce sont les denrées les plus valorisées du moment. Ce déplacement des denrées vise à informer le visiteur que la famille est en difficulté et à justifier l'accueil simple qui lui est offert. Soulignons à nouveau cette démarche particulière d'orientation symbolique en fonction de la manière dont on veut se donner à voir.

23 Dans le secteur zegün se trouve le lit conjugal, et c'est assis à son chevet (zegün qojtu)que le chef de famille accueille ses invités, alors que sa femme s'assoit au pied du lit, côté zegün uridu. Entre le pied du lit et la porte, se trouve l'espace consacré à la préparation de la nourriture. Il comporte un meuble d'une hauteur d'un mètre environ, où sont rangés les aliments et la vaisselle. Des réserves d'eau et de combustible sont parfois intercalées entre la porte de la yourte et ce meuble. Cet espace est essentiellement fréquenté par les femmes, puisque ce sont elles qui se chargent de nourrir le feu et la famille(Bianquis-Gasser 1996).

24 Pour finir ce tour d'horizon, dirigeons-nous vers le poêle situé au centre de la yourte. En hiver, on utilise plus volontiers un poêle en métal d'un seul tenant (à rayonnement calorifique plus important), de forme cylindrique, alors qu'en été, on fabrique un poêle en briques. L'ouverture du foyer est orientée soit vers la porte, soit vers le pied du lit des maîtres de maison. Ce choix se justifie tant sur le plan fonctionnel que sur le plan symbolique puisque, au moment où la maîtresse de maison prépare le repas, elle manipule alternativement nourriture, eau et combustible qui se trouvent dans ce secteur ; cette ouverture marque aussi la place qu'elle se doit d'occuper le reste du temps.

\section{Orientation et points cardinaux}

Les termes que nous venons de voir sont généralement traduits en français par nos points cardinaux, référents absolus et invariables, et par les notions de gauche/droite/ devant/derrière. Mais en mongol, ils sont employés pour différencier les secteurs de la yourte et ne renvoient pas systématiquement à une orientation unique ${ }^{21}$. On observe que, quelle que soit l'orientation absolue de la yourte, le secteur proche de la porte s'appelle uridu (traduit par sud ou avant), celui du fond qojtu (traduit par nord ou arrière) et que les côtés sont respectivement nommés baraүun et zegün (traduits par ouest et est ou droite et gauche). Des zones collatérales sont aussi distinguées : barayun uridu,zegün qojtu, etc.

26 L'exemple des Ordos, auquel il est souvent fait référence à propos d'orientation mongole, le montre d'ailleurs clairement, puisque leurs yourtes sont tournées vers l'est (Mostaert 1968, p.7) et que le terme barayun, traduit en général par "ouest ", désigne, chez eux, le sud. Si leur porte est en effet tournée vers l'est et que ce secteur, comme nous 
l'avons dit, se nomme uridu, alors la partie barayun de la yourte se trouvera effectivement orientée au sud.

Fig. 1 - Correspondance théorique entre points cardinaux et secteurs de la yourte

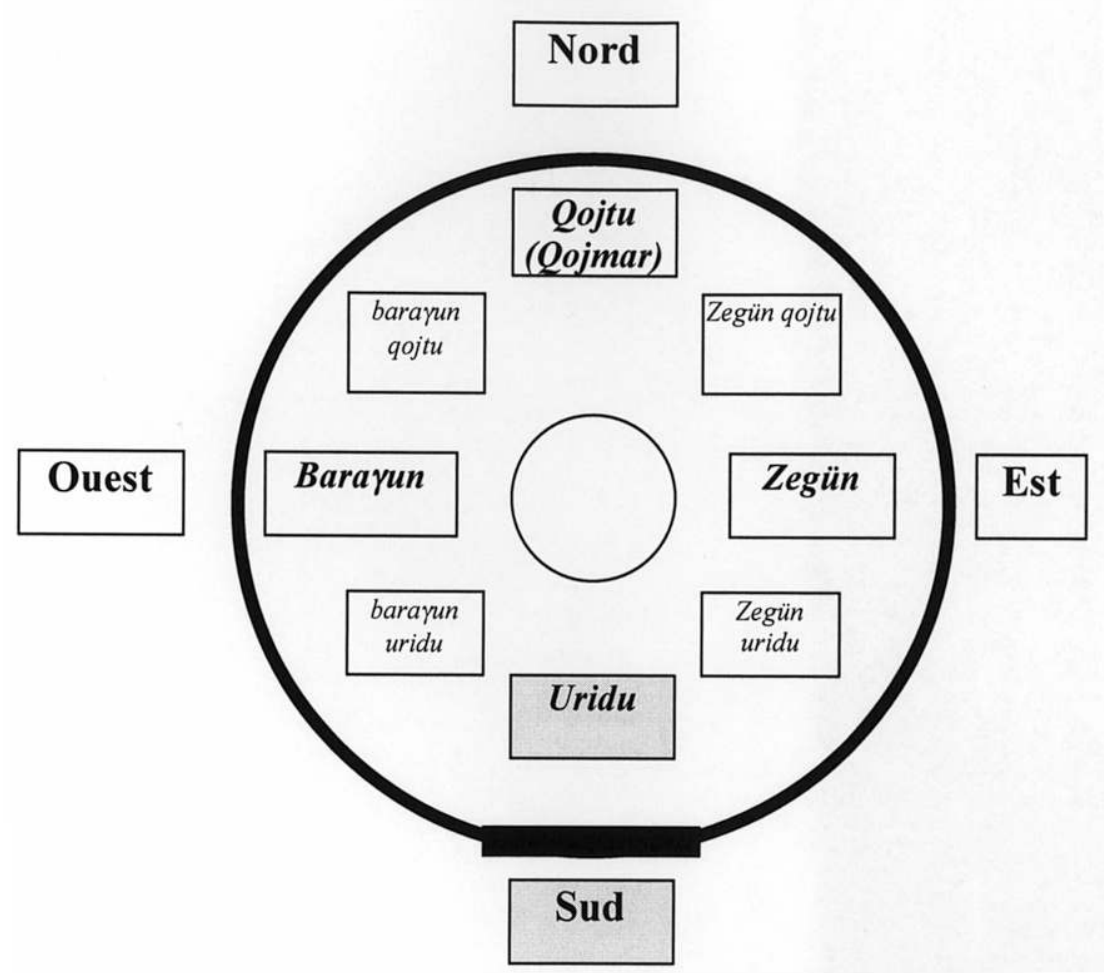

Fig. 2 - Exemple Ordos

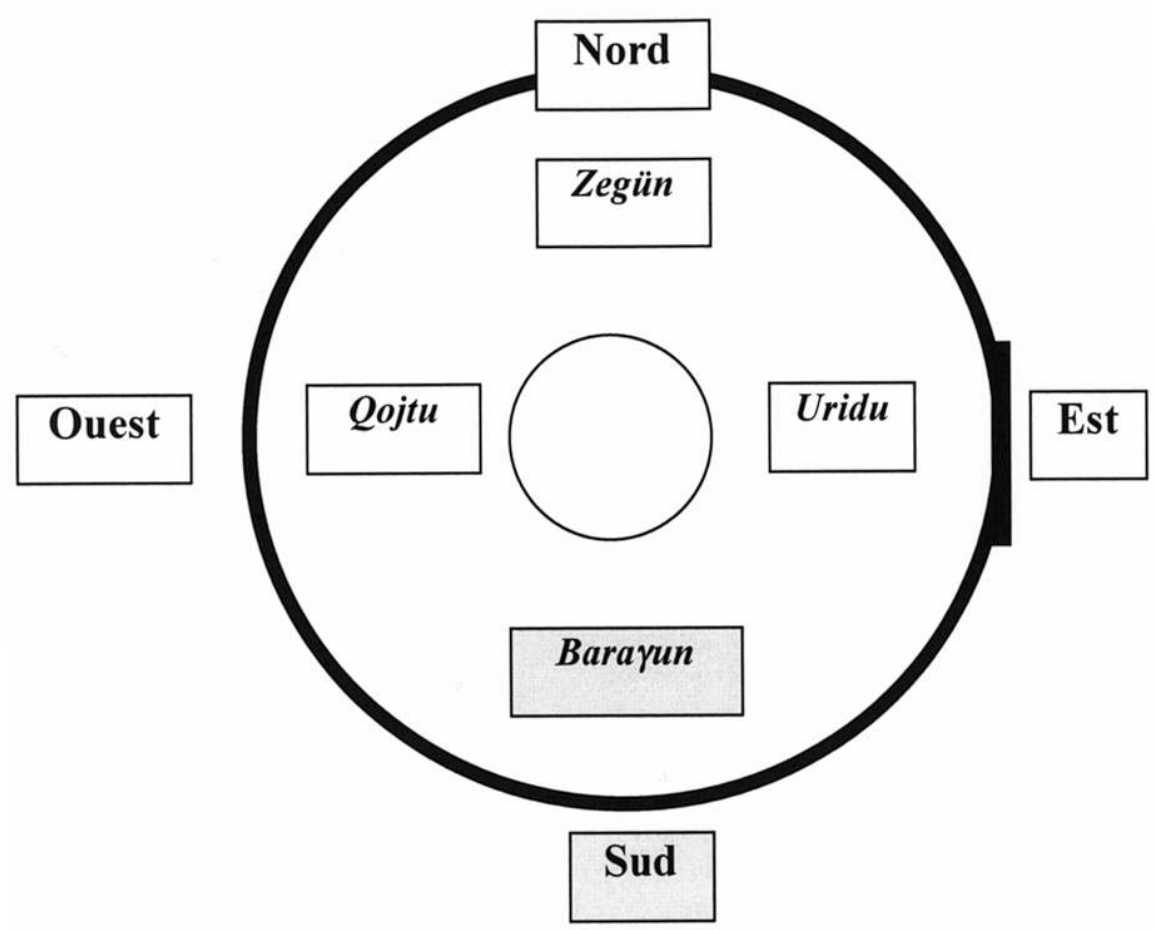


27 Dans la mesure où, hors de la yourte, les gens utilisent le terme : barayun pour désigner le sud, on peut poser l'hypothèse que c'est à partir du modèle de la yourte, luimême inspiré de celui du corps, que sont nommés les points cardinaux absolus. M.L. Beffa et R. Hamayon (1983, pp. 81-119) proposent qu'« en fait, si les termes sont les mêmes, c'est parce qu'ils expriment toujours l'orientation du corps humain face au soleil ; et si leur dénotation cardinale varie, c'est parce que le soleil est considéré tantôt à son lever, tantôt à sa culmination. On en revient donc toujours à une primauté chronologique de l'orientation relative - celle du corps - qui se figerait en orientation absolue - celle des points cardinaux - de deux façons différentes, face à l'est ou face au sud. Elles se concrétisent d'ailleurs dans les orientations respectives des portes des yourtes de ces populations » (Beffa et Hamayon 1983, p. 109). La dénotation ne varie-telle pasparce que c'est à la yourte qu'il est fait référence, et que - du moins dans une périphérie proche - c'est son modèle et sa terminologie qui sont étendus à l'environnement extérieur?

\section{Organisation extérieure : le campement}

28 Au-delà de la yourte, l'unité de vie domestique est le campement. Tout comme pour l'organisation intérieure de l'habitation, on note - à quelques variations près et quel que soit le nombre de yourtes ${ }^{22}$ - des manières similaires d'agencer le matériel d'un campement à l'autre. La disposition des tentes les unes par rapport aux autres informe généralement sur l'ordre de séniorité de leurs occupants. Elles sont la plupart du temps alignées et la yourte située approximativement le plus à l'ouest appartient à la famille aînée. D'ouest en est, l'ordre de séniorité décroît. Cet ordre quasi-immuable n'est troublé que par la présence ponctuelle de constructions stables comme l'enclos.

29 Derrière chaque yourte, on trouve d'abord l'éolienne puis sur ses abords, voire accolés à sa paroi, sont placés les outils (fourches, pelles, seaux, etc.), les bassines, et l'on y gare les motos. À une distance d'environ cinq mètres, les charrettes surmontées d'un coffre, alignées les unes derrière les autres, marquent une limite du campement. Elles contiennent des vêtements, des outils, de la nourriture et l'une d'elles porte le réservoir d'eau. Leur nombre varie en fonction de celui des yourtes. En moyenne, une famille possède trois coffres, un réservoir d'eau monté sur roues et une charrette qui sert aux déplacements quotidiens. Au-delà, on distingue une zone-débarras dans laquelle sont entassés des débris de bois et de métal qui sont emportés lorsque l'on nomadise et servent à dépanner en cas de besoin.

30 À environ cinq mètres en avant de la yourte, légèrement de côté pour ne pas gêner le passage vers la porte, se trouve le tas de combustible, puis à une dizaine de mètres de là, le poteau d'attache des chevaux ${ }^{23}$.

31 Quant aux enclos à bétail, nous avons observé toutes les dispositions possibles, variant en fonction de la présence ou non d'installations stables. On note seulement qu'on rapproche les enclos des yourtes au moment de l'hiver.

De manière générale, il est important de maintenir ordre et propreté devant la yourte. La place des motos, des tracteurs, des tas de combustible témoigne d'ailleurs de la bonne tenue du campement et $\mathrm{du}$ bon fonctionnement des activités au sein de la famille. En revanche, on accorde une attention moins grande à l'arrangement de l'arrière, où sont disposés les carrioles, les débris de bois, de métal et de vieilles 
carcasses de moto. S'agissant de l'extérieur, il se dégage donc une opposition entre le devant et l'arrière de la yourte.

\section{Repères relatifs, repères absolus}

Face aux différents agencements intérieurs et extérieurs, il ne semble pas possible d'associer des valeurs aux points cardinaux, mais on peut en attribuer à des secteurs. On note qu'à l'intérieur, la partie privilégiée est le fond, soit la partie qui se trouve devant soi en entrant. C'est là qu'est installé l'invité et que s'assoit le maître de maison. À l'extérieur, c'est au devant de la yourte qu'est porté le plus d'attention. Par conséquent, c'est la position de l'homme dans des situations définies qui est signifiante et, partant, ce qui se trouve devant lui est privilégié.

Nous avons vu que le recours aux points cardinaux ne permettait pas de rendre compte intégralement de la réalité changeante de la disposition des individus et des objets dans la yourte. C'est pour cette raison que, pour comprendre l'orientation des espaces habités par les éleveurs, nous penchons clairement pour la prise en compte de référents relatifs (corporels ou domestiques). Au-delà de la sphère domestique, on passe à un champ indépendant, dans lequel on se repère grâce aux points cardinaux géographiques. On peut ainsi distinguer trois référents majeurs : l'homme entrant dans la yourte, la yourte (chacun avec sa gauche, sa droite, son devant et son arrière), puis l'environnement lui-même, soumis à une orientation absolue.

\section{Mobilité et sédentarité}

35 Dans le discours commun, il est courant d'opposer nomadisme et sédentarité, mais la réalité est fort différente : il n'existe pas seulement deux formes opposées, mais des situations intermédiaires. En effet, le fait que des éleveurs occupent à un moment donné un bâtiment en dur ne signifie pas qu'ils se sédentarisent, car souvent ils n'habitent cette résidence que de manière temporaire.

36 Un bâtiment en dur est en général construit par une famille ${ }^{24}$ qui y séjournera pendant une période variable en fonction des activités pastorales du moment, des opportunités de travaux complémentaires, de ses stratégies économiques, etc. Elle est toujours amenée à le quitter temporairement, car les conditions environnementales imposent encore au minimum un déplacement annuel. Pendant ce temps, le bâtiment sera soit laissé vide, soit loué, soit encore prêté en échange de services. Mais au fil des années, la conjoncture évolue, les familles se réunissent, se divisent, changent d'activités et il est nécessaire de pouvoir toujours s'adapter à de nouvelles situations. C'est pourquoi les maisons passent de main en main, se négocient, se louent, se prêtent, tombent en ruine, se reconstruisent, sans jamais être habitées tout au long de l'année par une seule et même famille. Une maison est un édifice, à un endroit, à un moment, potentiellement disponible pour quiconque, en fonction des rapports entretenus avec les habitants précédents, les constructeurs, les acheteurs ou toute personne qui a séjourné en ce lieu et qui possède un droit temporaire à son égard. Ce phénomène renvoie à la question du droit d'usage propre aux éleveurs par opposition au droit de propriété caractéristique des agriculteurs ${ }^{25}$. 


\section{Le bâtiment en dur}

\section{Origines, composantes, formes et matériaux}

37 On le nomme baising, terme dont l'étymologie reste sujette à discussion: c'est sans doute un emprunt au chinois, peut-être à baixing (le peuple, littéralement cent familles), réinterprété ensuite dans le langage mongol courant, puis retranscrit à nouveau au XVI $\mathrm{e}^{\mathrm{e}}$ siècle par les Chinois sous la forme bansheng (Charleux 1997, p. 30). Ces derniers en auraient aujourd'hui oublié la signification originelle. On trouve aussi le terme baishang, pour désigner les premières installations sédentaires des Chinois installés sous les ordres et la protection d'Altan-khan (Jagchid et Hyer 1979, p. 313). Quoi qu'il en soit, l'usage mongol actuel veut que ce terme désigne un bâtiment en dur, quels que soient sa forme et ses matériaux, par opposition à la yourte.

À partir du $\mathrm{XVI}^{\mathrm{e}}$ siècle, des émigrés chinois s'installent peu à peu en MongolieIntérieure, en vagues importantes à partir du XIXe siècle. Ils développent l'agriculture auprès des Mongols et construisent des maisons sédentaires, des villages, des villes, apportant avec eux des techniques, des savoirs et des modèles chinois. Ce premier pas marque l'ancrage d'une influence architecturale qui va croître avec le temps. C'est surtout au $\mathrm{Xx}^{\mathrm{e}}$ siècle, à partir des années soixante, lors de l'apparition des communes populaires, que le rapport à l'habitat des éleveurs est profondément transformé. Certaines familles sont sédentarisées de force et la fréquentation régulière de bâtiments fixes est imposée à la population entière. Que ce soit pour des réunions, des recensements, des soins médicaux ou par le biais de l'école, peu à peu, les familles de pasteurs vont s'imprégner de nouvelles manières d'habiter.

Dans les années qui suivent, avec la poursuite de la colonisation chinoise et les problèmes posés par une surpopulation croissante, l'occupation de territoires jusqu'alors habités par des Mongols est aussi profondément bouleversée. En effet, sous la pression du chômage et de la famine, de nombreuses familles han quittent la Chine centrale et septentrionale pour venir s'installer dans la steppe, encouragées par le gouvernement central qui voit dans cet exode un moyen d'assimiler en profondeur les ethnies minoritaires. Les administrations locales de Mongolie-Intérieure sont chargées de réduire les parcours de nomadisme, de geler les frontières et de prendre en charge les nouveaux venus. Considérée par les Chinois comme un eldorado, la steppe se peuple d'agriculteurs, d'ouvriers et de marchands han qui ouvrent des petits commerces et achètent quelques têtes de bétail qu'ils confient à des familles de pasteurs mongols en échange d'un salaire. Ils parviennent ainsi à vivre bien plus aisément qu'en Chine propre.

40 Tout éleveur aspire aujourd'hui à se construire un bâtiment en dur, afin de participer à la modernité ambiante et de tenter d'adopter des stratégies économiques nouvelles ${ }^{26}$, quitte à n'y passer qu'une partie de l'année. Un bâtiment est dit plus confortable, plus chaud, plus pratique. Mais, de fait, l'espace plus restreint de la yourte est plus facile et plus économique à chauffer, et les éleveurs ne se privent pas de s'y installer aux mauvais jours, à proximité de leur bâtiment en dur ${ }^{27}$. Cette contradiction entre le discours et la pratique révèle que l'habitat en dur est un «signe extérieur de richesse » qui introduit une nouvelle forme de hiérarchisation sociale.

41 À la différence de ce qui se passe en ville, dans la steppe, les familles construisent leurs maisons de manière à ce qu'elles soient éloignées les unes des autres, pour 
bénéficier de pâturages étendus (Hurelbaatar 1996, p. 76), de sorte qu'il leur faut être auto-suffisants.

42 Rappelons que lors de la construction de bâtiments, les Mongols font appel à des Chinois han. Ils insistent d'ailleurs souvent pour dire qu'ils sont incapables d'en construire eux-mêmes, que seuls les Han le peuvent ainsi que les Mongols du Jirim (région au sud-est du Hulunbuir, dont la majorité s'est sédentarisée depuis bien longtemps). L'équipe d'ouvriers comprend donc toujours au moins un Han qui dirige les travaux à partir de plans établis par le propriétaire. Malgré l'influence technique han, nous allons voir que le modèle agricole chinois est adapté à certains critères propres aux éleveurs mongols et à leur mode de vie.

43 La forme des bâtiments rencontrés est très variable, il est donc impossible de proposer ici un modèle uniforme. Les bâtiments, couvrant une surface de 15 à $80 \mathrm{~m}$, sont soit parallélépipédiques - bien que deux exemples de bâtisses rondes aient été relevés - soit composés de cellules juxtaposées les unes aux autres, sans aucune règle apparente quant à leur agencement mutuel. Les murs, de trois mètres de hauteur, sont pour la plupart montés en briques rouges ou en adobe ${ }^{28}$. Par-dessus, on applique un mélange de terre et de paille, parfois de la chaux. On trouve également un grand nombre de maisons en pisé29. Elles sont ensuite couvertes d'une charpente faite de minces tasseaux de bois, elle-même recouverte de papier, de chaux, puis de paille mélangée à de la terre. Les fenêtres, qui ne peuvent pas s'ouvrir, font un mètre carré et sont divisées en six vitres. Elles sont orientées préférentiellement vers le sud. Les portes d'entrée, en bois, sont recouvertes d'une couche de tôle fine ${ }^{30}$ et comportent une serrure ou un cadenas. Elles sont le plus souvent dirigées vers lenord, mais cette orientation reste variable. Toutes ces maisons sont de plain-pied et pour la plupart munies d'éoliennes qui alimentent une ampoule et éventuellement une radio.

\section{Organisation intérieure}

44 L'habitat en dur est au minimum composé d'une cuisine et d'une pièce séparée dans laquelle se trouve un $\mathrm{kang}^{31}$. La cuisine se trouve généralement du côté le moins ensoleillé, soit au nord de l'habitation. Toutefois, de manière systématique, c'est par cette pièce que l'on s'introduit dans la maison. Elle est composée d'un foyer en briques à deux ouvertures, d'un ou deux meubles et éventuellement d'une table si la surface au sol le permet. Dans ce cas, c'est dans cette pièce que le visiteur est reçu et invité à s'asseoir.

45 L'organisation globale de la pièce principale est conditionnée par la disposition du kang, relié au foyer en brique de la cuisine par un conduit dans le mur. La disposition du couchage et l'orientation du chevet du lit dépendent du nombre de personnes qui y dorment. Ainsi, la tête peut se trouver indifféremment au sud, au nord, à l'est ou à l'ouest. Les meubles - dont certains sont maintenant destinés uniquement à la maison en dur du fait de leur hauteur - sont disposés de manières très variées. Dessus, sont posées la machine à coudre, les valises etc. Les plus beaux meubles, ainsi que tous les objets qui les surmontent - qui composent le nouveau secteur honorifique du bâtiment - se trouvent en général face à la porte d'entrée. Si l'organisation de la pièce ne le permet pas, ils sont disposés de manière à être en vue du visiteur, c'est-à-dire en face ou à côté de la place qui lui est réservée, située sur le kang ou sur une chaise face à la table. On note également une tendance à vouloir surélever au maximum le support 
des objets prestigieux : trois à quatre valises sont empilées sur un meuble, et au sommet de cette pile sont disposés les bibelots. Ils peuvent ainsi se trouver à un mètre cinquante du sol. Il en est de même des cadres de photos de famille, qui sont de ce fait parfois difficiles à contempler.

\section{Organisation extérieure}

46 À la différence des campements nomades, l'organisation extérieure n'est pas uniforme, les aménagements variant selon les familles. Les éléments qui persistent sont les charrettes surmontées d'un coffre en métal ou d'un réservoir d'eau, l'éolienne, le tracteur, l'outillage, les bassines et quelques vieux essieux servant à tanner le cuir. Parmi les équipements qui apparaissent avec le bâtiment, on observe des pompes à eau et de petits ateliers qui offrent de nouvelles possibilités de stockage (d'eau, de nourriture et de matériel).

Malgré le relatif « désordre » qui ressort de cette libredisposition, une constante se dégage, concernantl'une des composantes majeures de la zone extérieure à la maison : les coffres en métal qui contiennent du matériel précieux ${ }^{32}$ sont toujours laissés à portée de vue de la fenêtre de la principale pièce de vie (cuisine ou salle de réception). Dans un campement nomade, ils se trouvent au dos de la yourte de sorte que, la nuit, la tête tournée dans cette même direction, les habitants peuvent entendre les bruits suspects. Les parois du bâtiment constituant désormais une barrière au son, il devient alors nécessaire de pouvoir surveiller ses biens d'une autre manière : c'est à la vue qu'il est fait appel.

\section{Les transformations}

Ainsi, en passant de la yourte au bâtiment, les changements principaux relèvent de l'extension de la surface au sol et de la hauteur, de l'apparition d'angles et de parois droites, du nombre de pièces, du choix des matériaux, de l'orientation et du mode d'aménagement. Ces transformations engendrent des ruptures, mais présentent aussi des continuités. Pour comprendre les premières, nous analyserons tout d'abord l'impact de la division en différentes pièces et de l'utilisation de nouveaux matériaux sur les rapports des hommes entre eux et avec l'environnement extérieur. Puis, nous éclaircirons certains mécanismes de base qui guident l'orientation domestique et ses transformations.Nous en dégagerons les rationalités communes afin de saisir les éléments qui gardent un sens pour les éleveurs dans leurs manières d'habiter en général.

\section{Ruptures}

\section{Repère}

La première rupture que l'on peut noter est le transfert du repère majeur d'orientation par rapport aux points cardinaux. Dans la yourte, la porte marque la direction générale de l'habitat et elle est orientée approximativement vers le sud. En revanche, dans le bâtiment, ce sont les fenêtres de la pièce principale qui sont orientées dans cette direction. C'est donc l'ouverture la plus lumineuse qui devient le repère de 
l'orientation, et non plus la porte qui, dans le cas du bâtiment, peut être orientée dans toutes les directions possibles.

\section{Masculin/féminin}

50 Alors qu'il augmente la surface d'habitation, le bâtiment scinde l'espace en plusieurs pièces et cette multiplication a tendance, entre autres, à transformer la répartition sexuelle qui avait cours dans la yourte. La cuisine reste un espace plutôt féminin puisque c'est la maîtresse de maison qui $\mathrm{y}$ règne. Seulement, la construction de cet espace défini, cloisonné, sépare la ou les femmes du reste de la famille. Les hommes et les invités ${ }^{33}$ se réunissent dans la pièce principale: si celle-ci n'est pas la cuisine, hommes et femmes de la maisonnée partagent moins les conversations. Les murs tendent alors à cantonner les différentes catégoriessociales dans des espaces relativement hermétiques, aspect inexistant dans la yourte caractérisée par son espace unique. Les compartiments, créés au sein même de l'habitat, entraînent ainsi une nouvelle spécialisation de l'espace par la création de nouvelles distinctions socialesau sein de la famille. Les enfants, les adultes, les femmes et les hommes tendent à se regrouper entre eux et à avoir de moins en moins d'interactions les uns avec les autres.

\section{Le mur : division et distanciation}

51 Dans l'habitat en dur, au-delà de la cuisine ou de la zone principale d'accueil, il peut exister d'autres pièces séparées par des parois. Parfois, les portes donnant sur des chambres personnelles sont fermées par des cadenas, de manière à en interdire l'accès à l'étranger ou aux autres membres de la famille elle-même. Une sphère privée et personnalisée apparaît et introduit un sentiment nouveau d'appartenance et d'individualité. Toutefois, il ne faut pas oublier que, dans les maisons composées d'une seule pièce, ce sentiment se révèle tout à fait absent. C'est donc la division rendue possible par l'extension de l'espace habitable qui instaure ce changement dans les rapports sociaux.

En outre, les différents matériaux utilisés dans la yourte et le bâtiment sont le reflet de deux cultures aux modes de subsistance distincts. Les Han, peuple d'agriculteurs, se servent de la terre pour édifier leurs maisons alors que les Mongols, peuple d'éleveurs, utilisent le feutre. Au premier abord, ceci induit déjà un rapport à l'environnement radicalement différent. La yourte présente des propriétés adaptées à l'élevage mobile : un espace restreint plus facile à chauffer grâce au combustible disponible, une articulation des parois qui laisse passer les bruits pour être à l'écoute du troupeau et une légèreté qui la rend plus facile à transporter. Dans le cas du bâtiment, le mur de brique et ses fondations créent une étanchéité plus grande aux bruits extérieurs qu'une couche de feutre posée sur des treillis à même le sol. Par ailleurs, l'apparition des fenêtres dans le bâtiment offre un regard différent sur le monde environnant. Nous avons montré de quelle manière l'agencement extérieur des objets est dicté en partie par un souci de surveillance: le mur de la maison devient une barrière au son, la fenêtre joue donc un rôle nouveau qui privilégie la vue. Les matériaux ont ainsi des conséquences sur des attitudes telles que la protection et l'orientation des hommes et des choses à l'intérieur et à l'extérieur de l'habitat.

53 D'autres transformations apparaissent dans les comportements, liées au souci de se protéger. Une attitude d'apparence anodine a souvent attiré notre attention : avant de 
se coucher, que ce soit dans la yourte ou dans un bâtiment, il est d'usage que chacun sorte faire ses derniers besoins. Dans le cas de familles qui séjournent dans un bâtiment, l'arrivée de la nuit apporte un sentiment de peur qui oblige chaque habitant à sortir accompagné et muni d'une lampe torche. Une grande angoisse se manifeste à l'idée d'ouvrir la porte. De retour à l'intérieur, c'est avec soulagement que chacun se rassoit et évoque les risques encourus. Cette appréhension trouve son expression dans un type de discours que nous n'avons jamais eu l'occasion d'entendre dans une yourte. De celle-ci, au contraire, on sort plus facilement seul, même si les femmes s'invitent facilement entre elles pour partager rires et cachotteries ${ }^{34}$. Il n'y a pas non plus autant d'attention concentrée autour du moment où l'on ouvre la porte. La disponibilité d'esprit permet aussi d'observer le ciel et les étoiles, de sentir le vent, afin de prévoir le temps du lendemain. La rupture entre le monde du dedans et du dehors est bien moins marquée. Cette différence peut être illustrée aussi par le fait que la porte d'un bâtiment est fermée à clé la nuit alors que celle de la yourte est habituellement laissée ouverte. Pour finir, notons que, dans la yourte, au moment du coucher, les personnes qui s'installent au sol de manière transversale entre les deux lits orientent leur tête vers la montagne sacrée la plus proche ${ }^{35}$. En aucun cas les pieds ne seront orientés dans sa direction. Dans le bâtiment, ce type de critère d'orientation n'est plus guère invoqué. Ces différents aspects révèlent clairement des changements de perception de l'autre et de l'environnement qui s'opèrent lors du passage de la yourte au bâtiment.

\section{Continuités}

54 La récurrence de l'organisation d'une yourte à une autre et toute la symbolique qui l'accompagne ont une importance fondamentale d'un point de vue social. Cet ordre implique un positionnement réfléchi des choses et incarne un consensus accepté localement de façon à se (s'y) « reconnaître ». Cette forme normative d'orientation est déconstruite et recomposée dans le bâtiment, dans lequel n'apparait pas de schéma uniforme. Cependant, une cohérence propre au bâtiment se met en place en fonction de la disposition des murs, du kang, de la zone honorifique, de la place du visiteur, etc. Toutefois, en comparant les deux espaces domestiques, l'on voit apparaître des règles communes. Ces persistances permettent de mieux comprendre certains fondements de leur appréhension de l'environnement écologique et social.

\section{Le feu}

Les pasteurs mongols considèrent le feu du foyer comme un élément protecteur et purificateur. Il est sacré, personnifié et respecté de telle sorte qu'il ne faut pas y jeter de déchets, ce qui risquerait d'attiser sa colère. Or il en est de même dans un bâtiment, malgré la décentralisation du foyer. La première pièce dans laquelle on accède en entrant est invariablement la cuisine, passage obligatoire pour qui s'introduit dans la maison, et lieu où se trouvent les foyers en briques. Cette disposition que l'on retrouve systématiquement dans tous les bâtiments habités par des Mongols, suppose une motivation très forte derrière la volonté de placer la cuisine à l'entrée de la maison. En effet elle ne se rencontre pas dans l'architecture chinoise han où la cuisine est située soit à l'extérieur, soit à l'intérieur, mais toujours au fond. En tout cas, jamais elle ne constitue la pièce par laquelle on s'introduit dans l'habitat, ni celle dans laquelle on reçoit. L'aspect pratique d'un accès plus facile au combustible se trouvant dehors est 
bien entendu non négligeable, mais nous sommes ici en présence d'un exemple intéressant de choix architectural délibéré, dicté par le respect d'une conception très ancrée du feu comme protecteur et purificateur de l'espace domestique, propre aux éleveurs mongols.

\section{La place du visiteur} communication entre les groupes est fondamentale. Elle permet, entre autres, de régler la répartition de l'accès à l'eau, aux pâturages et aux parcours, afin d'assurer une coordination adéquate des déplacements et des activités, un équilibre et une entente entre les groupes. C'est pourquoi on attend beaucoup du visiteur, porteur de nouvelles ; et si on le traite avec égard, c'est aussi pour s'assurer un bon accueil lorsqu'on se déplacera soi-même. rôle clé qu'il joue au sein du groupe, le visiteur joue également un rôle décisif dans l'orientation et la disposition des individus et des objets. Lorsqu'il s'introduit dans la yourte, il fait tout d'abord face à la zone honorifique. Celle-ci lui permet d'évaluer le niveau social de la maisonnée et de se comporter en conséquence. Puis, l'endroit où il se place et la manière dont il est reçu informent du statut du visiteur vis à vis de la famille qui l'accueille. Nous avons vu que l'espace d'un bâtiment est différemment agencé, notamment vis-à-vis du visiteur. Celui-ci est accueilli dans la cuisine et, si elle n'est pas suffisamment spacieuse, dans la pièce de réception. Dans la cuisine, il est invité à s'asseoir de préférence sur une chaise, (près de la table et, en hiver, à proximité du poêle), c'est-à-dire sur une place en hauteur. Néanmoins, si les visiteurs sont nombreux, la répartition des sièges se fera selon les mêmes règles que dans la yourte, fondées sur l'opposition aîné/cadet et masculin/féminin. On attribuera donc au visiteur le plus prestigieux la place estimée être la plus confortable et celle où il sera le moins amené à se déplacer. De plus, la zone honorifique qui se trouve dans la salle de réception est, comme dans la yourte, située dans le champ de vision de l'invité, c'est-àdire face à la porte de la pièce, mais jamais contre le mur attenant à celle-ci.

L'orientation des individus et des objets est donc fortement influencée, dans le bâtiment en dur comme dans la yourte, par la nécessité de bien accueillir le visiteur et, surtout, de lui permettre d'évaluer correctement le statut de la famille par le moyen d'objets exposés dans le secteur honorifique de la maison.

Cependant, avec la récente modernisation des moyens de transport et la réduction croissante des parcours de nomadisme, on commence à observer des changements dans les pratiques d'hospitalité. Désormais, la moto, le tracteur et le $4 \times 4$ permettent une plus grande mobilité et ainsi une multiplication des sources d'informations. Alors qu'il y a quelques années encore, le caractère exceptionnel et primordial des visites garantissait la qualité de l'accueil, la perte d'importance du visiteur en tant que porteur de nouvelles transforme les manières de recevoir. Elles tendent à se réduire et à devenir beaucoup plus sélectives, en fonction de l'intérêt que l'on porte à recevoir telle ou telle personne.

60 Ces changements transforment les usages, mais ne vont-ils pas également modifier l'organisation interne de l'habitat dans lequel le visiteur jouait naguère un rôle si important? 


\section{Conclusion}

61 Le présent travail se donnait pour objectif de comprendre les changements dans l'occupation de l'espace domestique entraîné par le passage de la yourte au bâtiment en dur et, par delà, de déterminer les fondements des choix en matière d'orientation d'un groupe d'éleveurs mobiles. Considérant que l'organisation intérieure et extérieure de l'habitat reflète l'importance accordée aux différents aspects de la vie et aux manières de percevoir la réalité, nous avons voulu dégager les aspects majeurs des transformations. Nous avons ainsi pu voir que le passage de la yourte à un bâtiment en dur modifie le rapport à l'environnement et à l'Autre, entraîne l'apparition de formes d'intimité nouvelles et exclusives, transforme, par le cloisonnement, la répartition des espaces réservés aux hommes et aux femmes, et implique la prédominance de la vue sur l'ouïe dans la surveillance du bétail ou des biens matériels. En revanche, nous avons aussi pu observer que l'importance de la protection offerte par le feu perdure, ainsi que les dispositions spatiales mises en œuvre pour l'accueil des visiteurs, même si certains changements de disposition préfigurent l'introduction de nouvelles échelles de valeur.

62 En outre, il est apparu que l'habitat fonctionne comme une sorte de boussole sociale. Sa disposition et son aménagement sont en partie dictés par des représentations d'un ordre idéal, variable dans le temps. Cela n'est donc pas tant l'orientation par rapport à un orient qui est essentiel à l'intérieur de l'habitat, car s'orienter en ce cas, ne se réduit pas à s'adapter à une configuration ou à se conformer à une norme symbolique absolue. Orienter les choses et les êtres dans l'habitat, c'est à la fois se positionner par rapport à quelque chose et parler de soi, décliner une identité. C'est aussi se conformer à un modèle capable de situer l'Autre, au contact duquel une codification spécifique se met en place. L'orientation interne prend ainsi la forme d'une grille symbolique flexible.

\section{BIBLIOGRAPHIE}

Aubin, F.

1986 Le folklore comme mass media : l'exemple de la selle chez les Mongols, L'Ethnographie, LXxxII, 98-99, pp. 119-144.

Beffa, M.-L. et Hamayon R.,

1983 Les catégories mongoles de l'espace, Études mongoles et sibériennes, 14, pp. 81-111.

Bianquis-Gasser, I.

1996 La gauche et la droite. Principe de répartition des hommes et des femmes en République de Mongolie, Revue des Sciences sociales de la France de l'Est, 23, pp. 99-103.

Bulag, U.

1998 Nationalism and Hybridity (Oxford, Clarendon Press). 
Charleux, I.

1997 Histoire et architecture des temples et monastères lamaïques de Mongolie méridionale.

Thèse de doctorat de l'Université de Paris IV-Sorbonne.

Hamayon, R.

1979 Des fards, des mœurs et des couleurs, in S. Tornay (éd.), Voir et nommer les couleurs (Nanterre, Laboratoire d'ethnologie et de sociologie comparative), pp. 207-247.

1990 La chasse à l'âme. Esquisse d'une théorie du chamanisme sibérien (Nanterre, Société d'ethnologie).

Humphrey, C.

1998 Rituals of death in Mongolia. Their implication for understanding the mutual constitution of person and objects and certain concepts of property, Inner Asia, I, 1, pp. 59-86.

Hurelbaatar, A.

1996 Herding with cultivating in Inner Mongolia, Inner Asia Occasional Papers, I, 2, pp. 69-90

Jagchid, S. et P. Hyer

1979 Mongolia's Culture and Society (Boulder, Colorado).

Kotwicz, W.

1929 Sur les modes d'orientation en Asie Centrale, Rocznik Orjentalistyczny (Lwów), 5, pp.

Mostaert, A. (CICM)

[1941] 1968 Dictionnaire ordos (Pékin, The Catholic University), 3 vol., 1941-1944, [Monumenta

Serica, 5].

Sneath, D.

2000 Changing Inner Mongolia, Pastoral Mongolian Society and the Chinese State (New York, Oxford University Press).

Vreeland, H. H.

[1954] 1962 Mongol Community and Kinship Structure (New Heaven, Human relations Area Files Press).

Wasilewski, J.

1976 Space in nomadic cultures, in W. Heissig (éd.), Altaica Collecta. Berichte und Vorträge der XVII. PIAC (3.-8. Juni 1974, Bonn) (Wiesbaden, Otto Harrassowitz), pp. 345-360.

\section{NOTES}

1. Ger en mongol, mais pour une plus grande facilité de lecture, nous opterons pour ce terme d'origine turque communément utilisé pour désigner la tente en feutre des éleveurs nomades. Les transcriptions utilisées ici sont celles du mongol littéraire commun aux écrits de toutes les ethnies mongoles.

2. Le présent travail se base sur des données récoltées en langue mongole entre 1996 et 2002 dans la région du Barga, du nom de la population mongole (issue de la branche mongole septentrionale et proche des Bouriates) qui l'occupe depuis le XVII ${ }^{\mathrm{e}}$ siècle. Elle est située dans la ligue (division administrative) de Hulunbuir en Région autonome de Mongolie-Intérieure (République populaire de Chine). À compter de sa mise en place au milieu du XVII ${ }^{\mathrm{e}}$ siècle, le Barga est tiraillé par les grandes puissances qui se disputent son territoire. La zone est d'abord soumise à la dynastie sino-mandchoue des Qing (1644-1911). À la chute des Mandchous et lors de la déclaration d'indépendance de la République de Mongolie en 1911, elle se rattache à cette dernière. Mais les Russes et les Japonais ayant déjà signé un traité de répartition de leurs zones d'influence, le Barga tombe finalement sous le contrôle du Mandchoukouo gouverné par les 
Japonais. En 1945, après la conférence de Yalta, les Japonais sont expulsés, et la région est à nouveau rattachée provisoirement à la République mongole. Puis en 1947, avec l'arrivée des troupes communistes, elle est reprise par la Chine et intégrée dans la Région autonome de Mongolie-Intérieure (RAMI), créée à cette même date.

3. On note ici l'apparition d'une nouvelle gamme de couleurs, introduite, entre autres, par le principal producteur local de yourtes, un Han. Jusqu'alors, les éleveurs mongols privilégiaient l'orange, le jaune et le bleu pour le mobilier et l'armature de leur yourte. L'introduction du rouge, du vert et du bleu foncé (mais aussi du gris et du rose clair pour les meubles) tend à transformer le paysage intérieur de la maison. Pour une étude approfondie sur l'usage et la signification des couleurs, voir Hamayon (1978).

4. Sur la question voir aussi Vreeland ([1954]1962, p. 147) et Sneath (2000, p. 194).

5. Xojmor, sous sa forme khalkha moderne.

6. Il faut environ 300 briques pour fabriquer un revêtement de sol.

7. Ü̈̈d, sous sa forme khalkha moderne.

8. Jadis, elle était simplement faite d'une couche de feutre.

9. La pratique locale veut que l'on s'introduise dans la yourte toujours avec le pied droit en premier, le contraire serait perçu comme une impolitesse. Ceci permet d'offrir une explication à la disposition des charnières à gauche.

10. Je note à cette occasion que, lors d'une courte enquête de terrain en Mongolie, j'ai pu noter l'absence de ce type de porte. Les yourtes possèdent des portes à un seul battant, sans fenêtre, et les habitants étaient très surpris à la vue de mes photos de Mongolie-Intérieure. Il est donc possible d'attribuer l'apparition de fenêtres sur les portes des yourtes des éleveurs de MongolieIntérieure à une influence typiquement chinoise.

11. Cf. Mostaert (1968), Jagchid etHyer (1979), Kotwicz (1929), Wasilewski (1976), Charleux (1997).

12. Du moins depuis la division du pays sous la dynastie des Qing (1644-1911).

13. Les Mongols un tant soit peu militants de Mongolie-Intérieure se plaisent à manipuler le symbole du cheval. On peut le trouver par exemple au-dessus des portes de leurs maisons, sous la forme d'une petite statuette de porcelaine représentant un buste de cheval qui se tord le cou pour se tourner vers la République mongole. Il serait ainsi devenu un signe discret de mécontentement individuel.

14. Sur le rôle, dans l'affirmation identitaire, de l'orientation de la selle sous la yourte, voir Aubin (1986).

15. Cette enquête de terrain fut menée en 2001 avec Y. Dorémieux.

16. Pour combustible, les éleveurs utilisent essentiellement du crottin de vache séché et des branches de bouleau.

17. L'outre était faite originellement d'une peau de bête cousue en forme de sac, mais l'objet dont nous parlons ici est en fait un sac en tissu dans lequel on laisse le fromage de vache s'égoutter.

18. On notera en effet que les éleveurs mongols se plaignent de leur santé fragile et l'attribuent au fait qu'ils dorment à même le sol, et que le froid qui s'en dégage fragilise leur ossature et occasionne des douleurs qui surviennent tôt. Cet argument revient souvent pour expliquer leur désir de construire une maison en dur.

19. On en trouve en effet souvent plusieurs et réglées à des heures différentes.

20. «Les ongon, destinés à servir de support à des esprits humains sont le plus souvent réalisés non comme des sculptures mais comme des tableaux, en deux dimensions : sur un rectangle de tissu, portant des croquis peints d'animaux, d'arbres et d'astres, et des silhouettes humaines, dessinées ou découpées dans une plaque de métal et appliquées [...]; s'il en est généralement ainsi pour des séries (par exemple pour des ancêtres), des esprits individuels peuvent avoir pour ongon des sortes de poupées » (Hamayon 1990, p. 771). 
21. À ce sujet, voir l'article de Kotwicz (1929, p. 83) qui montre combien les termes d'orientation mongols ne peuvent se réduire à l'équivalent de nos points cardinaux. On voit en effet qu'en fonction des groupes, les mêmes termes mongols renvoient à des orientations absolues différentes. "Les vocables barayun(>barun), «droit», "ouest», et žegün, [...], " gauche », « est » sont parfois utilisés avec la signification de «sud» et «nord ».. Ce fait est connu depuis longtemps ; en 1776 déjà, Pallas écrivait que zun (soon, " gauche ») désignait, chez les Kalmouks, le nord, bien qu'ils plaçassent leurs yourtes, la porte au midi : par conséquent, la terminologie n'était plus d'accord désormais avec la vie journalière) ».

22. Le nombre de yourtes dans un campement varie de un à cinq en fonction des saisons, des activités d'élevage et des fêtes.

23. Cet élément semble contraster avec la pratique des Mongols de Mongolie qui tendent une corde entre deux poteaux pour attacher leurs chevaux. Chez les Barga de Mongolie-Intérieure, il est aussi fréquent d'attacher son cheval aux charrettes derrière la yourte.

24. Les familles font tout de même appel à un spécialiste han.

25. Pour une recherche approfondie sur le concept de propriété chez les Mongols, se référer à l'article de Humphrey « Rituals of death in Mongolia » (1998), dans lequel, à travers les concepts de personne exprimés au moment des rituels funéraires, l'auteur identifie une relation de propriété propre aux éleveurs mongols.

26. Ces stratégies sont variées, mais elles peuvent consister en l'ouverture d'un magasin, d'une auberge ou encore en l'adoption d'une agriculture vivrière, laquelle nécessite une possibilité de stockage.

27. Dès la fin du XIx ${ }^{\mathrm{e}}$ siècle, on observe des exemples similaires : les princes se font construire des palais et les moines des monastères. Chacun y séjourne durant l'été et préfère la yourte durant l'hiver (communication personnelle d'Isabelle Charleux, 2002).

28. Brique d'argile non cuite, obtenue par simple séchage au soleil. Cette technique est d'ailleurs de plus en plus pratiquée par les Mongols eux-mêmes, alors qu'ils disent ne pas avoir assimilé celle de la construction.

29. Maçonnerie faite de terre pilée argileuse à laquelle on ajoute de la paille que l'on entasse entre des banches.

30. Quand elles sont claquées, ces portes font un bruit tout à fait différent de celles des yourtes. Leur sonorité est volontairement accentuée pour permettre d'entendre si quelqu'un entre ou sort de la maison.

31. Lit en brique chauffé par un conduit venant du foyer de la cuisine.

32. Ils contiennent des réserves de nourriture et des vêtements qui garantissent la survie de la famille en hiver, mais aussi des tissus, du feutre, des outils ainsi que tout un ensemble d'objets considérés comme vitaux.

33. Quel que soit leur sexe, même s'il est fréquent de voir les femmes venir aider et discuter en cuisine avec la maîtresse de maison.

34. Cette attitude vise aussi certainement à conjurer la peur, mais à un degré moins fort que dans le bâtiment.

35. On s'oriente vers l'une des deux montagnes sacréesenvironnantes, Boyda a pula ou

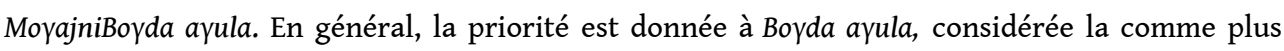
puissante. 


\section{RÉSUMÉS}

Actuellement, dans les zones de steppe de Mongolie-Intérieure, l'habitat des éleveurs nomades connaît des transformations majeures. Alors que jusqu'ici ils demeuraient majoritairement dans des tentes de feutre, ils s'installent aujourd'hui dans des bâtiments en dur, sans pour autant se sédentariser. L'étude comparative de l'orientation, d'une part de la yourte et du bâtiment, et d'autre part des individus et des objets à l'intérieur de chacun de ces habitats, révèle que le positionnement en fonction des points cardinaux absolus n'est pas primordial. Une conjonction de facteurs à la fois géographiques, religieux, symboliques et socio-économiques guide les manières de choisir des orientations spécifiques. Cet articlemet en évidence les ruptures et les continuités dans le passage de la yourte au bâtiment en dur et souligne le caractère flexible des représentations liées à une organisation idéale de l'espace domestique.

Nowadays, in grassland areas of Inner Mongolia, mobile herders' dwellings are going through major changes. Whereas previously most Mongol herders lived in felt tents, they are now settling in houses, without necessarily becoming sedentary. The comparative study of the external orientation of the dwellings and the orientation of individuals and objects within the dwellings, shows that their position is not determined primarily by cardinal points. A combination of geographic, religious, symbolic and socio-economic factors guide the ways of choosing specific orientations. This articlebrings to light the ruptures and continuities in the passage from the yurt tothe fixed dwellingand underlines the flexible character of representations related to an ideal organisation of domestic space.

\section{INDEX}

Index géographique : Chine, Mongolie, Mongolie Intérieure, Russie

Thèmes : habitat, orientation

nomsmotscles Barga, Bouriate, Chinois, Halh, Han

Mots-clés : environnement, hospitalité, identité culturelle, mobilité, sédentarité

Keywords : cultural identity, environment, hospitality, mobility, sedentary lifestyle

\section{AUTEUR}

\section{ALEXANDRA MAROIS}

Alexandra Marois est doctorante au sein du Laboratoire d'Ethnologie et de Sociologie Comparative de l'Université de Paris X-Nanterre. Elle s'intéresse aux transformations de l'habitat et aux échanges de savoirs entre Chinois Han et Mongols de Mongolie-Intérieure. 